\title{
Médiévales
}

Langues, Textes, Histoire

73 | automne 2017

Le texte à l'épreuve du numérique

\section{Thomas LABBÉ, Les Catastrophes naturelles au Moyen} Âge

Paris, CNRS éditions, 2017, 352 p.

\section{Sarah Claire}

\section{(2) OpenEdition}

Journals

Édition électronique

URL : https://journals.openedition.org/medievales/8250

DOI : $10.4000 /$ medievales. 8250

ISSN : 1777-5892

Éditeur

Presses universitaires de Vincennes

Édition imprimée

Date de publication : 15 décembre 2017

Pagination : 246-247

ISBN : 978-2-84292-801-8

ISSN : 0751-2708

Référence électronique

Sarah Claire, "Thomas LABBÉ, Les Catastrophes naturelles au Moyen Âge », Médiévales [En ligne], 73 |

automne 2017, mis en ligne le 15 décembre 2017, consulté le 22 avril 2022. URL : http://

journals.openedition.org/medievales/8250; DOI : https://doi.org/10.4000/medievales.8250

Ce document a été généré automatiquement le 22 avril 2022.

Tous droits réservés 


\title{
Thomas LABBÉ, Les Catastrophes naturelles au Moyen Âge
}

Paris, CNRS éditions, 2017, 352 p.

\author{
Sarah Claire
}

\section{RÉFÉRENCE}

Thomas LABBÉ, Les Catastrophes naturelles au Moyen Âge, Paris, CNRS éditions, 2017, 352 p.

1 L'ouvrage de Thomas Labbé répond à l'appel ambitieux de Jacques Berlioz, lancé il y a vingt ans, de réaliser une histoire totale des catastrophes naturelles à la période médiévale. L'auteur complète la considérable historiographie en sciences sociales de l'objet « catastrophe » en privilégiant l'angle d'approche de l'histoire des sensibilités, auparavant délaissée. La démarche socio-anthropologique adoptée s'appuie sur un impressionnant corpus de 3146 documents, majoritairement des sources administratives, narratives et pastorales, répartis dans l'Occident médiéval du XIII ${ }^{\mathrm{e}}$ au $\mathrm{XVI}^{\mathrm{e}}$ siècle. L'intelligibilité de l'événement naturel extrême explorée dans le livre permet d'isoler le paradigme de rationalisation et de représentation des catastrophes spécifique à la période médiévale.

2 L'ouvrage se divise en deux parties thématiques. La première est consacrée à l'étude du système de représentation à l'origine de la perception des phénomènes naturels dans la pensée médiévale. La deuxième partie porte sur les attitudes comportementales qui résultent du processus social de ritualisation de la catastrophe et révèlent la demande de sens de la société. Des tableaux statistiques ou de mise en regard des champs lexicaux, inclus dans le corps du texte, éclairent les textes sur lesquels s'appuie l'auteur. Ces deux parties sont précédées d'une préface de Jacques Berlioz et d'une longue et riche introduction proposant un point historiographique approfondi sur le traitement de la catastrophe en sciences sociales. Parmi les annexes, l'auteur a réalisé un index des événements naturels très utile au lecteur. 
3 Premièrement, l'auteur analyse dans le discours médiéval l'imaginaire d'appréhension des catastrophes naturelles qui s'articule autour du couple émotionnel casus-signum, proche de notre distinction contemporaine aléa-catastrophe. L'étude de cette grille d'analyse, enrichie par l'examen du vocabulaire conceptuel d'une sélection de sources narratives, aboutit à un modèle fondé sur la notion de causalité et non de conséquence. L'auteur montre ainsi la spécificité du paradigme médiéval qui joint des phénomènes destructeurs et d'autres sans conséquences matérielles ni humaines (p.41). Deux significations sont attribuées aux phénomènes naturels : une fonction purificatrice et une fonction augurale, qui trouvent toutes deux leur origine dans l'attitude pécheresse des hommes. L'étude de ces deux schémas explicatifs permet à l'auteur de poser les premières bases, bien peu renseignées jusqu'à présent, d'une géographie et d'une sociologie des peurs, en se fondant sur les liens de causalité établis par les auteurs médiévaux entre certains comportements humains et certaines catastrophes naturelles (p. 149). Ces dernières ne retiennent pas le même niveau d'attention chez les auteurs médiévaux qui opèrent une hiérarchisation, non pas en fonction de la gravité de leur impact socio-économique, mais selon le degré de compréhension des causes de l'événement. Par exemple, les comètes sont les événements les plus souvent dotés d'une signification par les chroniqueurs, malgré l'absence de conséquences humaines et matérielles (p.125). Ce schéma de raisonnement, fondé sur la complémentarité entre explications théologique et naturelle, perdure jusqu'à la fin du Moyen Âge. À partir du $\mathrm{Xv}^{\mathrm{e}}$ siècle, la place des phénomènes terrestres aux conséquences socio-économiques dans le système de représentation s'accroît, au détriment, par exemple, des phénomènes cosmiques. En somme, «ce qui impacte fait désormais sens au même titre de ce qui interroge ", résume Thomas Labbé (p. 138).

4 Dans un deuxième temps, l'auteur analyse les réactions aux phénomènes naturels extrêmes induites par l'affectivité décrite dans la première partie. En multipliant les points de vue documentaires, Thomas Labbé montre la construction narrative d'une norme sociale qui repose sur trois thèmes descriptifs récurrents : échapper soi-même au danger immédiat, ne pas s'occuper des autres, implorer la miséricorde divine (p. 186). Le sentiment religieux et le souci spirituel se trouvent au centre de la demande des populations dans l'action d'urgence, alors que la sphère victimaire et le sentiment humanitaire ne participent pas au processus de reconstruction, chose surprenante pour des observateurs actuels. Après l'étude des premières réactions d'absorption émotionnelle, l'auteur aborde la deuxième étape du processus de reconstruction sociale, celle de l'encadrement des populations par les autorités, dont l'action est structurée par des présupposés émotionnels qui traduisent les lignes d'horizon mental de la société (p. 203). L'étude des modalités de gestion des catastrophes par les autorités met en évidence le peu d'attention portée à la situation matérielle des victimes, caractéristique des dispositifs de gouvernement de cette période, sinon pour assurer la subsistance des administrés en cas de disette ou octroyer un allégement temporaire de la fiscalité. L'attitude des autorités se modifie à partir des $\mathrm{XV}^{\mathrm{e}}-\mathrm{XVI} \mathrm{I}^{\mathrm{e}}$ siècles, la victime prenant une place de plus en plus importante dans le système de représentation des catastrophes naturelles.

5 Cette division en deux parties, le discours d'un côté et les attitudes de l'autre, qui permet de mettre en lumière leur cohérence respective, rend peu compte de la rupture identifiée par l'auteur dans l'évolution des sensibilités et des modes de gestion des catastrophes. Thomas Labbé voit dans la période des $\mathrm{Xv}^{\mathrm{e}}$-XvI ${ }^{\mathrm{e}}$ siècles un changement 
dans la perception de la catastrophe par les auteurs médiévaux dont l'intérêt se déplace des causes vers ses conséquences (p. 138). Le regard porté par les autorités sur la sphère victimaire et les attentes de l'ensemble de la population à cet égard s'intensifient dans le discours et les actions des contemporains. L'auteur explique ce déplacement par l'expansion des nouvelles valeurs politiques de l'humanisme qui recentrent les préoccupations et l'observation de la réalité sur l'homme (p. 240). Cette transformation des discours et de l'action vis-à-vis de la catastrophe accompagne la réforme des institutions d'assistance, l'évolution des mentalités envers les pauvres, l'entrée de l'aide aux victimes dans le traitement politique, etc. (p. 246). Ces nouveaux discours sur la catastrophe bénéficient de plus de visibilité grâce à deux nouveaux genres littéraires qui apparaissent au Xvi ${ }^{e}$ siècle, les occasionnels et les canards, diffusés en Europe par l'imprimerie (p. 266). Une nouvelle image en particulier apparaît, celle de la quantification du bilan humain et matériel causé par les catastrophes, en lien avec le développement des figures de la victime et du héros. L'événement catastrophique ne fait plus sens uniquement par la charge significative qu'on lui attribue, mais davantage par ses conséquences socio-économiques dramatiques et sa dimension tragique. Le champ sémantique de la pitié et de la terreur utilisé par les auteurs des $\mathrm{XV}^{\mathrm{e}}-\mathrm{XVI}^{\mathrm{e}}$ siècles (p. 273) remplace celui de la puissance et de l'étonnement chez les auteurs des siècles précédents (p. 55).

6 L'auteur participe et invite à davantage d'historisation des émotions de solidarité, de la souffrance humaine et de l'altruisme, ainsi que des figures de la victime et du héros. Il propose également une périodisation de la sensibilité aux catastrophes en trois temps : un âge du signe du XII ${ }^{e}$ au Xve siècle, un âge du désastre aux XVv-XVII ${ }^{e}$ siècles et un âge de la catastrophe à partir du XVIII ${ }^{\mathrm{e}}$ siècle, ce dernier ayant bénéficié d'un traitement historiographique abondant (p. 281). L'auteur replace ainsi dans une perspective historique plus longue les discours d'affect et de raison générés par les phénomènes extrêmes. La rupture entre les deux premiers âges, celui du signe et du désastre, provoquée par l'accroissement de la prise en considération de la sphère victimaire, est bien moins visible que celle marquant le passage à l'âge de la catastrophe au xVIII ${ }^{e}$ siècle. En effet, si les phénomènes violents continuent à être considérés comme des fléaux divins, le XVIII ${ }^{\mathrm{e}}$ siècle fait éclater le modèle religieux d'interprétation des phénomènes naturels (p.288). Thomas Labbé s'attache à replacer son objet de recherche dans l'évolution plus générale des sensibilités concernant la souffrance sociale et le rapport à la mort, ou la construction du statut moderne de victime, et donne ainsi une clé de lecture de la société médiévale dans son ensemble. 